\title{
Übersichtsartikel
}

\author{
Gundela Meyer*
}

\section{Vom lateralen Rand ins Zentrum des Kortex: Die Entwicklung der menschlichen Inselrinde}

https://doi.org/10.1515/nf-2018-0008

Zusammenfassung: Die menschliche Inselrinde ist ein Hauptknotenpunkt in einem neuronalen Netzwerk, welches aus dem eigenen Körper stammende Erregungen mit Einflüssen aus der Umwelt integriert und dabei die autonome, emotionelle und sozial-kognitive Homöostase des Körpers erhält. Die Insel ist in den letzten Jahren ins Zentrum der Aufmerksamkeit gerückt. Vergleichende anatomische Untersuchungen haben gezeigt, dass die Insel in vielen Spezies am lateralen Rand des Neokortex liegt. Wenig erforscht ist die vorgeburtliche Entwicklung der menschlichen Insel, die sich als frühestes Kortexareal entwickelt. Ursprung der Pyramidalzellen der Insel ist ein kleiner Abschnitt der proliferativen Ventrikulär/Subventrikulärzone an der kortikostriatalen Grenze (KSG). Von den hier liegenden Zellen der radialen Glia, die als Stammzellen dienen, entspringt ein dichtgewebtes Faszikel aus Gliafortsätzen, welches durch die Capsula externa läuft und als Migrationssubstrat für die Neuroblasten aus der KSG in die Insel dient. Um die 10/11 Schwangerschaftswoche biegen sich der Lateralventrikel und die ihm anliegende KSG C-förmig um, sodass sich die Insel nun zwischen einer dorsalen fronto-parietalen und einer ventralen, temporalen KSG entwickelt und von diesen mit absteigenden bzw. aufsteigenden Migrationsströmen von Neuroblasten entlang des radialen Gliafaszikels versorgt wird. Als Folge der Ventrikelrotation wird die menschliche Insel während der Ontogenese vom lateralen Rand des Kortex in dessen Mittelpunkt verlagert, was im Einklang mit ihren integrativen Funktionen innerhalb der Hirnaktivität steht.

Schlüsselwörter: Radiale Glia; neuronale Migration; Seitenventrikel; Rindenschichten; Ontogenese.

*Korrespondenzautor: Gundela Meyer, Facultad de Medicina, Universidad de La Laguna, Tenerife, Spanien, gundelam@aol.com

\section{Einführung}

Die Inselrinde (Insel, Lobus der Insula) wurde erstmals 1809 von Reil in der Tiefe der Fissura Sylvii (Sylvische Furche, Sulcus lateralis) beschrieben. In den letzten zwei Dekaden ist die Insel in den Fokus der Forschung gerückt, weil sie an unerwartet vielen Bereichen menschlichen Denkens und Fühlens teilnimmt, und weil neue bildgebende Verfahren es uns ermöglichen, diese Bereiche zu erforschen. Eine kurze Aufzählung der Funktionen, die der Insel zugeschrieben werden, mag einen Einblick in ihre Bedeutung geben: Autonome Wahrnehmung (Interozeption), viszero-sensible, viszero-motorische und vestibuläre Funktionen, motorische Assoziation, Somatosensation, Chemosensation, Hören, Sprache, Emotionen, Schmerzempfindung, Empathie, Mitfühlen mit dem Schmerz Anderer, Zeitsinn, Aufmerksamkeit, Motivation, subjektive Musikwahrnehmung, Ästhetische Bewertung objektiver und subjektiver Schönheit, soziales Bewusstsein, Entscheidungsvorgänge, Suchtverhalten, Lust, Ekel, Riechen und Schmecken (zusammengefasst von (Nieuwenhuys, R., 2012) und (Kurth, F. et al., 2010)). Diese unterschiedlichen Funktionen werden bestimmten Subregionen der Insel zugeordnet, wobei der Informationsfluss von posterior nach anterior gerichtet ist. Als Beispiel mag die posteriore Insel dienen, die bei Wärme und Kältewahrnehmung, Hunger und Durst, sensorischer Stimulierung und Schmerz aktiviert wird (Craig, A.D., 2010, 2011). Die anteriore Insel hingegen ergänzt die Repräsentation des eigenen Körpers mit subjektiven Gefühlen und sozialen Interaktionen. Während der eigene Schmerz die posteriore Insel aktiviert, wird das Mitleiden mit dem Schmerz Anderer in der anterioren Insel wahrgenommen (Singer, T. et al., 2004). Hierbei zeigen rechte und linke Insel unterschiedliche Aktivationsmuster: Die rechte anteriore Insel steht unter sympathischem Einfluss und wird von Gefühlen mit negativem emotionalem Wert aktiviert; die linke anteriore Insel wird dagegen mit positiven Emotionen und parasympathischer Erregung assoziiert. Beide Inseln arbeiten dabei komplementär, nicht nur in dieser, sondern auch vielen anderen Funkionen (Craig, A.D., 2011; Medford, N. und 
Critchley, H.D., 2010). Die Insel kalibriert kardiorespìratorische und kardiovaskuläre Erregungen als Antwort auf exterozeptive und interozeptive Einflüsse, wobei die anteriore rechte Insel eine wichtige Rolle spielt und als zentraler Knotenpunkt im interozeptiven Netzwerk dient. Als Ganzes integriert die Insel alle Einflüsse aus der Umwelt und aus dem eigenen Körper, um Überlebensstrategien zu entwickeln und gleichzeitig die emotionale und physiologische Homöostase zu erhalten (Oppenheimer, S. und Cechetto, D., 2016). Craig hat die menschlichen Insel als Teil des „empfindenden Selbst“ definiert (Craig, A.D., 2010). Hierbei ist die Insel nicht als „Sitz“ bestimmter Funktionen anzusehen, sondern als wichtiger Knotenpunkt (engl. hub) in einem funktionellen, dynamischen Netzwerk, das viele andere Kortexareale involviert.

\section{Wo liegt die Insel im menschlichen Gehirn?}

Die Inselrinde liegt im Fundus der Sylvischen Furche, vollkommen bedeckt von den Opercula (Operculum: kleiner Deckel) der frontalen, parietalen und temporalen Lobi. Sie ist daher nicht an der Hemisphärenoberfläche sichtbar. An ihrem rostralen Ende grenzt sie an präfrontale Areale, während ihr caudales Ende durch die Heschl'schen Windungen des auditorischen Temporalkortex definiert wird (Abb. 1A). Ein zirkulärer Sulcus (sulcus circularis anterior, superior und inferior) umgibt die anteriore, dorsale und posteriore Inselrinde und grenzt sie von den umgebenden Kortexarealen ab. An ihrer freien vorderen und basalen Oberfläche, dem Limen insulae, geht die Insel in den Paläokortex des primären olfaktorischen Kortex über, der seinerseits die laterale Grenze der Substantia perforata anterior bildet. In der Tiefe der grauen Substanz der Insel liegt ihre weiße Substanz, die Capsula extrema, die die Insel mit ihren Projektionszentren verbindet. Medial zur Capsula extrema schließt sich das Claustrum an, das in seiner Ausdehnung fast deckungsgleich mit der Inselrinde ist. Ähnlich deckungsgleich ist auch das Putamen, der äußere Teil des Nucleus lenticularis, welches vom Claustrum durch die Capsula externa abgegrenzt ist. Diese Lagebeziehungen sind wichtig für den chirurgischen Zugang durch die Sylvische Furche zu tieferen Hirnstrukturen, besonders im Hinblick auf die große Anzahl an wichtigen Blutgefäßen in dieser Region (hauptsächlich die Arteria cerebri media und ihre Hauptäste in der Sylvischen Furche). Dies kommt auch in den vielen ausführlichen Beschreibungen der Anatomie der menschlichen Insel zum Ausdruck (Naidich, T.P. et al., 2004; Tanriover, N. et al., 2004).
Zwischen Capsula extrema und Capsula externa liegt das Claustrum, dessen Zugehörigkeit zu einem Claustroinsulärem Komplex kontrovers ist. In vielen Mammaliern ist die Lagebeziehung zwischen Claustrum und Insel sehr unterschiedlich, und auch im Menschen ist die dorso-caudale Insel durchaus nicht von einem Claustrum unterlagert. In diesem Review nehmen wir keinen weiteren Bezug auf das Claustrum und halten uns an die Meinung, dass Insel und Claustrum beide abhängig sind von einer engen Lagebeziehung mit dem olfaktorischen Kortex, ohne dass sie funktionell oder ontogenetisch zusammengehören (Buchanan, K.J. und Johnson, J.I., 2011).

Makroskopische Anatomie, Zytoarchitektur und Konnektivität der menschlichen Insel

Die Insel wird in eine größere anteriore und eine kleinere posteriore Inselregion unterteilt, welche vom Sulcus centralis insulae abgegrenzt werden. Die vordere Insel weist für gewöhnlich drei kurze Gyri auf, manchmal auch einen kleinen akzessorischen Gyrus (Abb. 1A). Ihre Anzahl kann variabel ein, nicht nur zwischen verschieden Gehirnen, sondern auch zwischen beiden Hemisphären (Abb. 1B). Die hintere Insel wird von zwei langen Windungen gebildet. Alle Gyri konvergieren radiär zum vorderen und ventralen Inselpol hin. Im Menschen wird außerdem ein frontoinsuläres Übergangsfeld an der Grenze zum orbitofrontalen Kortex beschrieben (von Economo, C. und Koskinas, G.N., 1925). Dieses Feld ist reich an großen Spindelneuronen in der Schicht V, den ,von Economo-Zellen“. Von-Economo-Zellen kommen auch im anterioren cingulären Kortex vor und haben weitreichende axonale Projektionen (Allman, J.M. et al., 2011).

Die zelluläre Architektonik (Zytoarchitektonik) der Insel im menschlichen und nicht-menschlichen Primaten hat seit jeher das Interesse der Forscher geweckt. Schon von Economo und Koskinas (von Economo, C. und Koskinas, G.N., 1925) unterteilten die Inselrinde des Menschen in einen vorderen agranulären Teil und einen hinteren granulären Teil, wobei in der mittleren Insel Zwischenstufen unterschieden wurden. Der Terminus „granulär“ oder „agranulär“ bezieht sich hierbei auf die Anwesenheit, Breite und Zelldichte der granulären Schichten II und IV des 6-schichtigen Isokortex, wobei besonders die Schicht IV entscheidend ist. Viele andere Autoren haben die zytoarchitektonischen Inselfelder im Detail untersucht. Da in den verschiedenen Studien die resultierenden Kartierungen der Insel in mögliche Unterfelder stark variieren, verweisen wir auf ausführliche Revisionen und Metaanalysen (Nieuwenhuys, R., 2012; Kurth, F. et al., 2010). Eine allgemein akzeptierte Parzellierung der Inselrinde des Makaken und Menschen stammt von Mufson und Mesulam (Mesulam, M.M. und Mufson, E.J., 1985), 

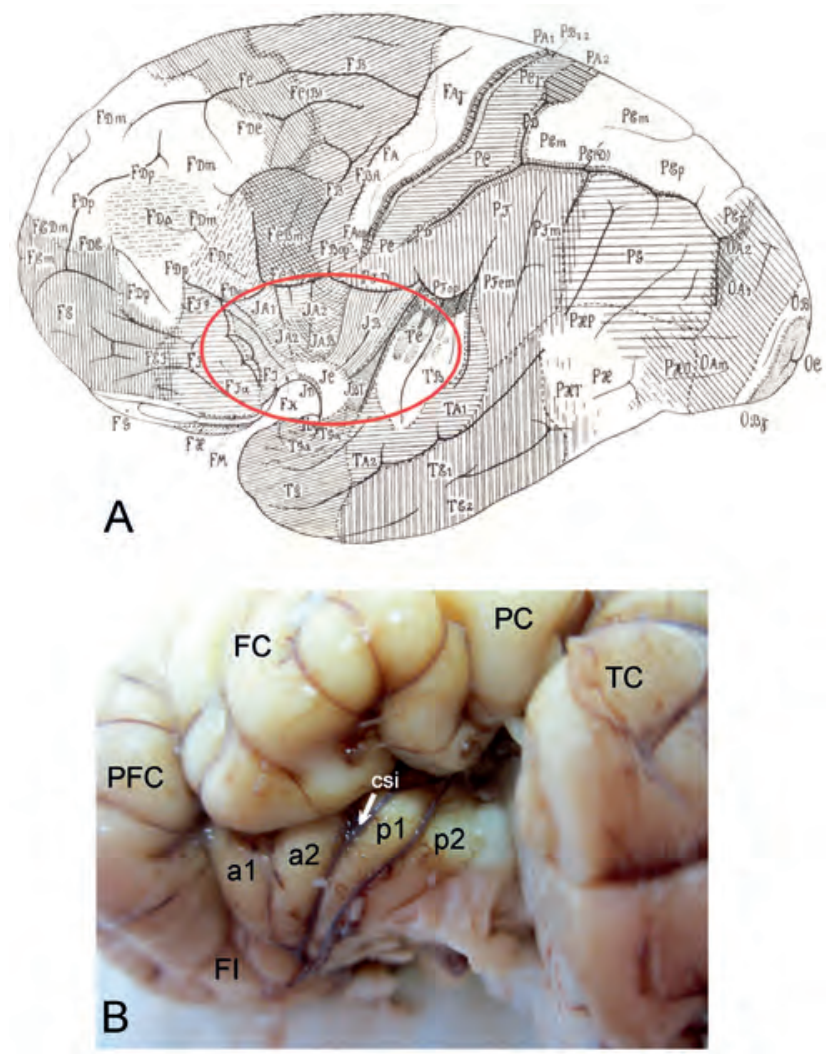

Abb. 1: A: Die Zytoarchitektur der lateralen Hemisphäre nach von Economo und Koskinas. Die Inselrinde in der aufgeklappten Sylvischen Furche ist rot umrundet. Man erkennt die zentrale Lage der Insel und ihre Grenzen mit den benachbarten Kortexarealen. B: Die Insel in einem Neugeborenen, das schon das definitive Gyrierungsmuster aufweist. Der vordere Pol des Temporallappens (TC) wurde entfernt, um die vorderen kurzen Gyri, in diesem Fall nur 2 (a1 und a2), und die beiden hinteren Gyri ( $\mathrm{p} 1$ und p2) in der Inselregion freizulegen. Der weiße Pfeil zeigt auf den Sulcus centralis insulae (csi), der rote Stern zeigt die Lage des Limen insulae an. FC: Frontaler Kortex; FI: Fronto-insuläre Rinde; PC: Parietalkortex; PFC: Präfrontalkortex.

obwohl weitere Unterteilungen durchaus möglich sind und auch vorgenommen wurden. Mufson und Mesulam unterscheiden eine vordere agranuläre Insel, in der eine Schicht IV nicht vorhanden ist, eine mittlere dysgranuläre Insel, in der eine Schicht IV unregelmäßig ausgebildet ist, und eine hintere granuläre Insel, die eine stark entwickelte Schicht IV aufweist. Wichtig ist der Umstand, dass die agranulären, dysgranulären und granulären Inselfelder fast konzentrisch von rostroventral nach caudodorsal um einen Orientierungspunkt herum angeordnet sind, der sich im vorderen und ventralen Inselpol befindet, und zwar dort, wo sich die Insel in den Paläokortex fortsetzt. Diese konzentrische Anordnung der Inselfelder gemäß der Ausbildung der Schicht IV wurde auch in anderen Spezies (Katze: 14, Ratte: 15) beobachtet, lässt sich aber durchaus

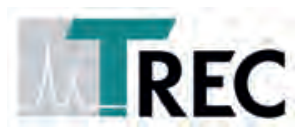

Thomas RECORDING GmbH

\section{In $\underline{\text { Cage Training System }}$ (ICTS) for Rodents}
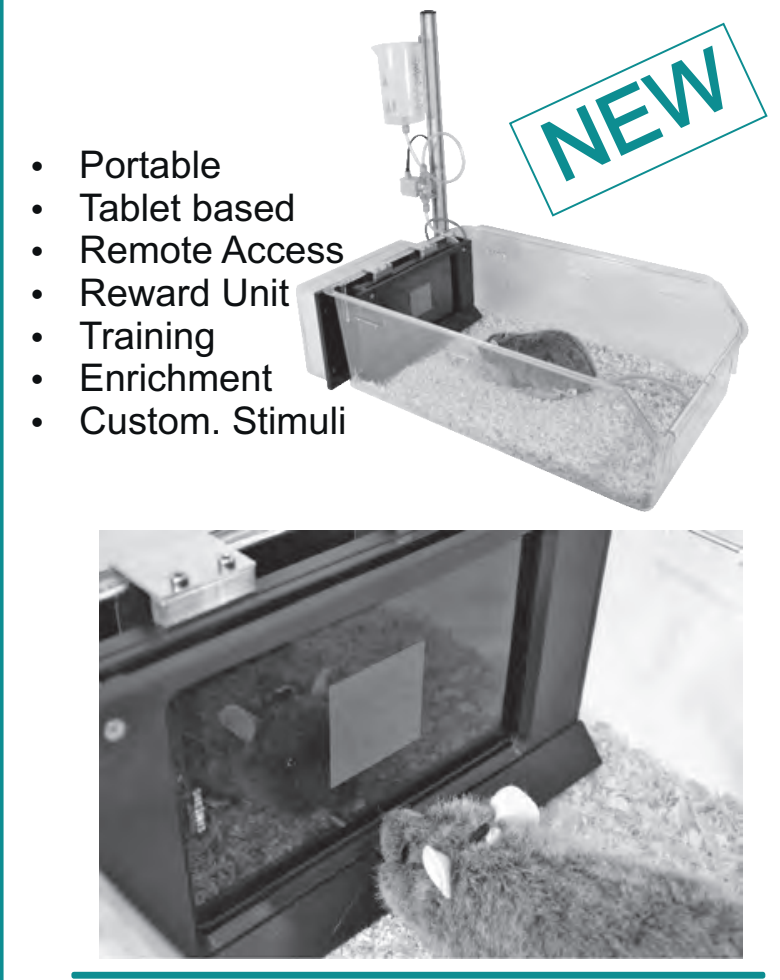

InCage Training System (ICTS) for NHP

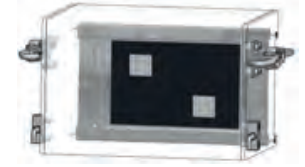

- Portable

- Tablet based

- Remote Access

- Reward Unit

- Training

- Enrichment

- Custom. Stimuli

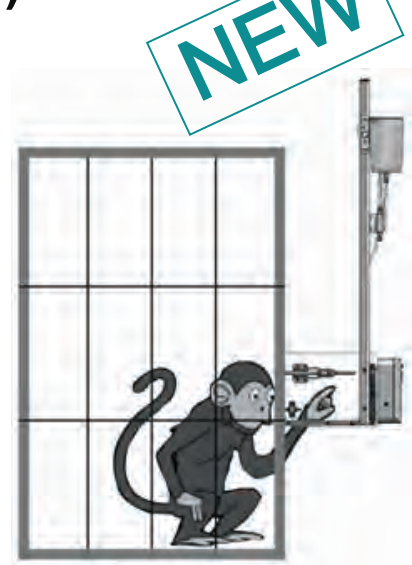

TREC @ 13th Göttingen Meeting of the German Neuroscience Society March 20 - 23 / Booth \# 5

Visit our website for more information: www.ThomasRECORDING.com 
nicht verallgemeinern (Butti, C. und Hof, P.R., 2010, siehe unten).

Die Konnektivität der Insel ist äußerst komplex. Viele der kortikalen und subkortikalen Verbindungen wurden in nicht-menschlichen Säugetieren beschrieben (Butti, C. und Hof, P.R., 2010; zusammengefasst von Nieuwenhuys, R., 2012; Augustine, J.R., 1996); erst die modernen Verfahren der Bildgebung ermöglichen die genaue Bestimmung der Faserverbindungen auch in der menschlichen Insel (Ghaziri, J. et al., 2017; Ghaziri, J. et al., 2018). Zusammenfassend lässt sich sagen, dass die Insel durch weitreichende kortiko-kortikale Verschaltungen mit praktisch allen Lobi vernetzt ist, auch mit weiter entfernten Rindengebieten im Okzipitalkortex und Entorhinalkortex. Besonders wichtig ist die gegenseitige funktionelle und strukturelle Verknüpfung mit dem cingulären Kortex, besonders dem anterioren cingulären Kortex, der ebenso wie die Insel von vielen kognitiven und affektiven Stimuli aktiviert wird (Medford, N. und Critchley, H.D., 2010). Weitere Verbindungen bestehen zwischen Insel und Hippocampus, Amygdala, Hypothalamus, Thalamus (inbesondere den Nuclei ventralis posterior inferior parvicellularis und ventralis posterior medialis parvicellularis, außerdem dem Nucleus dorsomedialis), aber auch zwischen Insel und Putamen, Globus pallidus, Nucleus caudatus und Nucleus accumbens (Augustine, J.R., 1996; Ghaziri, J. et al., 2018).

\section{Vergleichende Entwicklungsge- schichte der Inselrinde}

Die Insel wird allgemein als entwicklungsgeschichlich „alt“ und im engen Zusammenhang mit dem olfaktorischen Paläokortex und dem Claustrum betrachtet. Eine einflussreiche Theorie postulierte auf der Grundlage der gemeinsamen Expression von spezifischen Transkriptionsfaktoren in der proliferativen Ventrikulärzone während der Entwicklung, dass ein allgemeiner Bauplan der Hirnrinde (Pallium, Mantel), gültig für alle Amnioten ist (Puelles, L. et al., 2000). Dieser Bauplan hat vier Hauptregionen: 1) ein mediales Pallium, das den Hippocampus bildet, 2) ein dorsales Pallium, welches den zukünftigen Neokortex entstehen lässt, 3) ein laterales Pallium, das Insel, olfaktorischen Kortex und Claustrum bildet, und 4) ein ventrales Pallium als Ursprung der kortikalen Anteile der Amygdala. In Ratte und Maus liegt die Inselrinde als langgezogenes Band an der Oberseite des Sulcus rhinalis, wobei sie dorsal sukzessiv an den frontalen, parietalen und temporalen Kortex angrenzt, während sie ventral dem Paläokortex anliegt. Wie im Primaten lassen sich in der
Inselrinde der Nager agranuläre, dysgranuläre und granuläre Felder unterscheiden, die parallel zueinander von rostral nach caudal laufen (Fujita, S. et al., 2010). Auch in der Katze lassen sich agranuläre, dysgranuläre und granuläre Felder in Teilen des Sulcus rhinalis und des Gyrus orbitalis nachweisen (Clascá, F. et al., 1997). Mit dem Erscheinen der Sylvischen Furche und der Ausbildung des Temporallappens ventral zur Insel im Primaten ändert sich das Grundschema der Topografie der Inselrinde und der angrenzenden Lobi: Die Insel verlagert sich vom lateralen Rand des Kortex in dessen Zentrum.

Ist die menschliche Insel besonders prominent, was Größe und Struktur angeht? Dieser Frage sind Bauernfeind et al. (Bauernfeind, A.L. et al., 2013) nachgegangen, indem sie das Volumen der agranulären, dysgranulären und granulären Insel in 30 verschiedenen Primaten, einschließlich des Menschen, vermaßen. Tatsächlich ist der absolute allometrische Größenunterschied zwischen Mensch und Schimpansen in der linken und rechten agranulären Insel und dem linken frontoinsularem Kortex signifikant, und sogar stärker ausgeprägt als der Unterschied im präfrontalen Kortex. Diese Daten sprechen dafür, dass die anteriore Insel für soziales Bewusstsein und komplexe soziale Interaktionen wichtig ist, was im Falle des Menschen möglicherweise bedingt ist durch eine erhöhte Fähigkeit zur Empathie und zum Einfühlen in den Gefühlszustand Anderer.

Ein ganz anderer Aspekt der vergleichenden Anatomie der Insel wurde von Butti und Hof (Butti, C. und Hof, P.R., 2010) angesprochen. Sie untersuchten die Zytoarchitektur der Insel sehr unterschiedicher Spezies, die normalerweise nicht im Labor eingesetzt werden, wie z. B. Belugawal, Manatee, verschiedene Artiodactyla, und als Vertreter der Karnivoren das Atlantische Walross. In diesen Spezies ist die Inselrinde sehr unterschiedlich angelegt, lässt aber in keinem Fall die agranuläre, dysgranuläre und granuläre Organisation erkennen, die typisch nicht nur für Primaten, sondern auch für Ratte, Maus und Katze ist. Die Autoren weisen darauf hin, dass viele Spezies gar keine granuläre Schicht IV aufweisen, weder in der Insel noch in irgend einem anderen Areal. Ein weitergehendes Verständnis für die Evolution des Kortex, nicht nur der Inselrinde, sollte sich daher nicht auf Studien in den üblichen Laborspezies beschränken, sondern auch andere, alternative Modelle der Kortexentwicklung in Betracht ziehen. 


\section{Die Ontogenese der menschlichen Insel}

Angesichts der enormen Anzahl von makroskopischen, mikroskopischen, hodologischen und funktionellen Studien, die sich mit der Insel befassen, ist es erstaunlich, wie wenig wir über die vorgeburtliche Entwicklung der menschlichen Insel wissen. Eine erste Vertiefung einer Fossa Sylvii, zusammen mit dem ersten Auftreten der arteria cerebri media, erscheint in der 13. Schwangerschaftswoche (SW). Der Sulcus centralis insulae erscheint um die 18-21. SW, die weiteren insularen Sulci bilden sich um die 27-28. SW. Dabei ist die rechte Insel früher entwickelt als die linke (Afif, A. et al., 2007). Wichtig ist, dass Kortexentwicklung und Vaskularisierung der lateralen Hemisphäre ihren Ausgangspunkt in der Inselregion haben, die daher die ontogenetisch älteste Kortexanlage repräsentiert. Interessanterweise ist die Insel Ursprung früher neuraler (EEG) und hämodynamischer (fMRI) Spontanak- tivität, die sich als sogenannte „delta bursts“ schon mit 32-36 SW nachweisen lässt (Arichi, T. et al., 2017). Diese transitorischen Delta-Wellen im Niedrigfrequenzbereich, kombiniert mit hochfrequenten Alpha-Beta-Spindeln, breiten sich von der linken Insel und der rechten Insel plus Temporalkortex auf andere Kortexbereiche aus und verschwinden um die Geburt herum. Sie werden als Ausdruck des vorgeburtlichen Reifegrades des Kortex interpretiert. Die Lokalisierung der „delta bursts“ in der Insel zeigt an, dass diese eine Schlüsselrolle in der Reifung des menschlichen Gehirns spielt.

Die Frage, wie sich eine komplexe heterogene Struktur wie die menschliche Insel bildet, wurde von uns (González-Arnay et al., 2017) gestellt. Im Allgemeinen hat jedes Rindengebiet eine proliferative Ventrikulär- und Subventrikulärzone, aus der die postmitotischen Pyramidenzellen entlang der radialen Gliafortsätze zur Oberfläche migrieren, um sich in ihre endgültige Schicht im wachsenden Kortex eingliedern. Nach der „protomap“ Hypothese (Rakic, P., 1974) wird eine in der Ventrikulärzone vorgege-

\section{FiberOptoMeter II FOM-02M/FOM-02D}

Optogenetic Stimulation and Fluorescence Measurement via the Same Fiber Now available with 2 Detectors and Exchangeable Filter Cubes
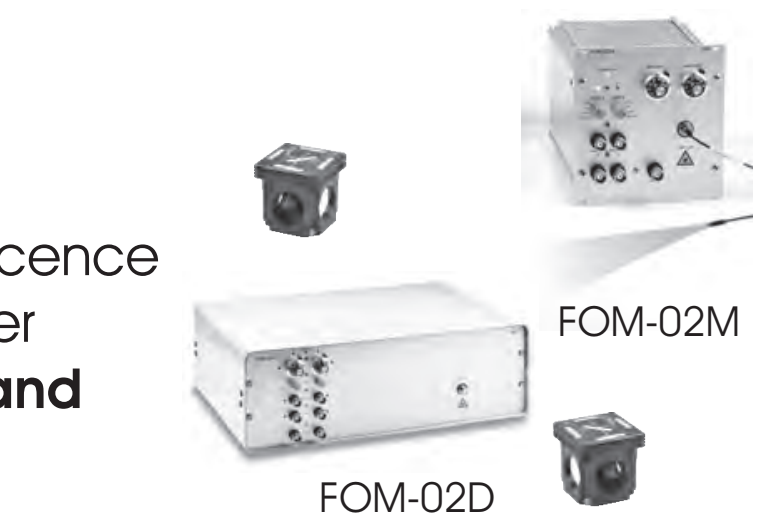

OGB-1 Fluorescence Measurement via a $200 \mu \mathrm{m}$ Fiber

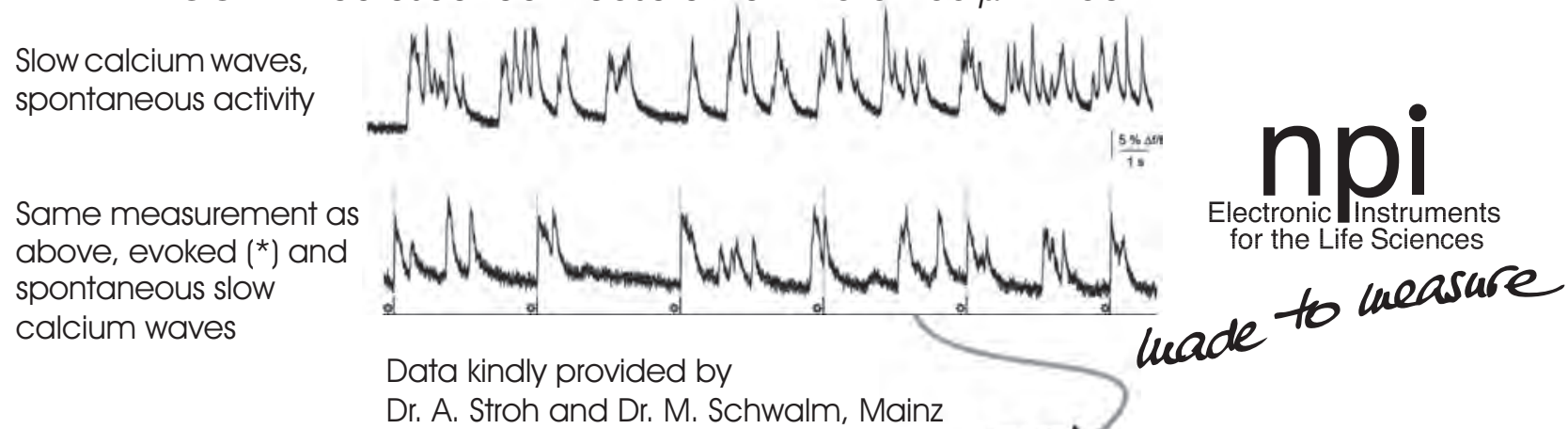

REF: Justus et al. (2016), Nat. Neurosci. http://dx.doi.org/10.1038/nn.4447

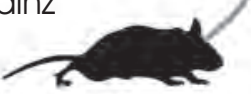

Adelsberger et al. (2014), Cold Spring Harb Protoc. http://dx.doi.org/10.1 101/pdb. prot0845

Stroh et al. (2013) Neuron, http://dx.doi.org/10.1016/j.neuron.2013.01.031

Phone: +49-(0)7141-97302-30

http://www.npielectronic.com support@npielectronic.com 
bene, primordiale „Protokarte“ positional in Bezug auf regionale Identität auf den sich entwickelnden Kortex übertragen. Im Gegensatz zu den benachbarten Lobi verfügt die Insel nur über eine sehr beschränkte Ventrikeloberfläche, die genau an der kortikostriatalen Grenze (KSG) liegt. Die immunohistochemische Anfärbung der radialen Glia mittels spezifischer Antikörper (gegen Vimentin oder Nestin) erlaubte uns eine klare histologische Darstellung der Fortsätze der radialen Glia, die sich fächerförmig von ihrem Ursprung in der Ventrikulär- und Subventrikulärzone ausbreiten und mit ihren Endfüßen bis an die piale Oberfläche reichen. Diese radiale Glia-Architektur, kombiniert mit Pallium-spezifischen Antikörpern wie Tbr1, zeigt den Migrationsweg der zukünftigen, Tbr1-positiven Inselneuronen von der KSG in die Insel (Abb. 2A). Als Migrationssubstrat dient ihnen ein enggewebtes Faszikel aus den Fortsätzen der radialen Glia, das durch die Capsula externa läuft (Abb. 2B, Abb. 3) und sich dann graduell in der Inselrinde verzweigt (González-Arnay et al., 2017).
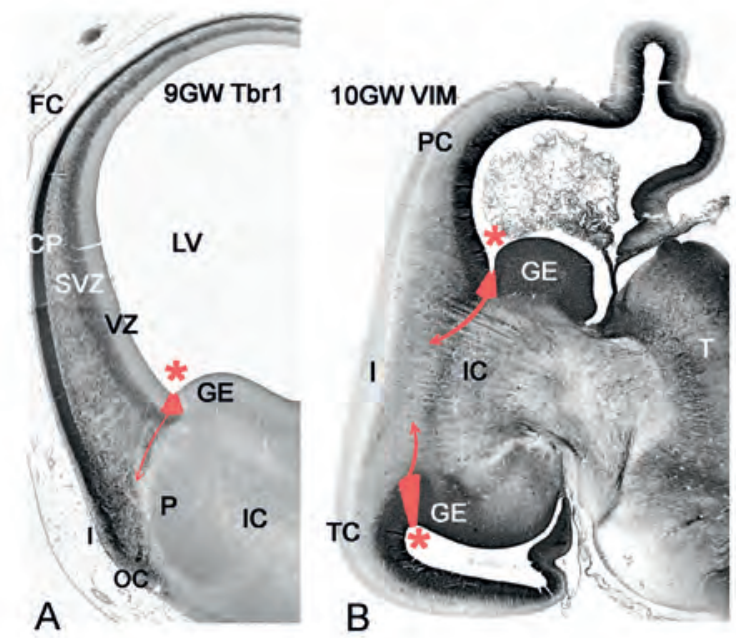

Abb. 2: A: Coronalschnitt durch ein Fetalhirn mit 9 SW. Der kortikale Teil des Telencephalons ist mit einem anti-Tbr1 - Antikörper immunohistochemisch angefärbt und zeigt die korticostriatale Grenze (in rot ihre Ventrikulärzone, Stern). Die Richtung des lateralen kortikalen Migrationsstroms in die Insel und den benachbarten Paläokortex ist mit einem roten Pfeil angedeutet. CP: kortikale Platte, FC: Frontalkortex, I: Insel, IC: Interne Kapsel, GE: Ganglionäre Eminenz, LV: Lateralventrikel, OC: Olfaktorischer Kortex, P: Putamen, SVZ: Subventrikulärzone, V: Ventrikulärzone.

B: Coronalschnitt auf einem etwas weiter posterior gelegenen Niveau eines $10 \mathrm{SW}$-alten Fetus, angefärbt mit einem Anti-VimentinAntikörper. Durch das Wachstum des Temporallappens ist die korticostriatale Grenze sowohl dorsal als auch ventral zu sehen. Die Insel liegt zwischen den dorsalen Frontal/Parietallappen und dem ventralen Temporallappen, und die Migrationsströme in die Insel folgen dem radialen Glia-Faserbündel, welches von der KSG abgeht (rote Pfeile).
Während der Fetalentwicklung definiert die KSG die Trennlinie zwischen den Rindengebieten und den Ganglienhügeln. Letztere sind subkortikale Strukturen, die die Basalganglien bilden, aber auch GABAerge Interneuronen für den Kortex hervorbringen. Die KSG wurde auch als „Anti-Hem“ bezeichnet, als ein mit bestimmten Entwicklungskontrollgenen (insbesondere Pax 6) angereichertes mögliches Signalzentrum, welches zusammen mit dem „kortikalen Hem“ am medialen Rande des Telencephalons die Regionalisierung des Neokortex instruiert (Assimacopoulos, S. et al., 2003). Eine evolutionäre Bedeutung der KSG wurde darin gesehen, dass die laterale Grenze des Palliums der Ursprung des „lateralen kortikalen Zellstroms" ist, welcher Neuroblasten in die Zentren führt, die dem lateralen und ventralen Pallium zugehörig sind: Der olfaktorische Kortex, der Endopyriforme Nucleus, das Claustrum und die kortikalen Anteile der Amygdala (Molnár, Z. und Butler, A.B., 2002). Ferner trägt die KSG zum sequenziellen Eintritt von thalamo-kortikalen und kortiko-thalamischen Fasern in die Capsula interna bei (González-Arnay et al., 2017; Molnár, Z. und Butler, A.B., 2002). Da die Definitionen des Anti-Hems und der KSG von Mammaliern (Rodentia) und Sauropsiden (Vögel und Reptilien) abgeleitet wurden, ist es nicht erstaunlich, dass der Insel in diesen Modellen keine weitere Bedeutung geschenkt wurde; tatsächlich ist die Inselrinde der Maus sehr unauffällig und erscheint eher als ventrales Anhängsel des lateralen Neokortex.

In der Untersuchung von González-Arnay et al. (González-Arnay et al., 2017) geben wir der KSG eine andere Deutung: Sie ist hauptsächlich der Ursprung der menschlichen Insel. Es ist bekannt, dass das Ventrikelsystem im Menschen sehr verschieden von dem der Nager ist. In den kleinen ungefurchten Nagergehirnen ist der Seitenventrikel nur leicht nach caudal und ventral abgebogen. Im Gegensatz dazu ist der menschliche Seitenventrikel C-förmig umgebogen, wobei sich schon früh in der Fetalentwicklung ein Cornu inferius im Temporallappen erkennen lässt. Während mit $9 \mathrm{SW}$ der Kortex glatt und eher nagerähnlich ist (Abb. 2A), erscheint mit 10/11 SW der laterale Ventrikel im sich entwickelnden Temporalkortex (Abb. 2B). Im Coronalschnitt mit 10 SW erscheint die KSG praktisch spiegelbildlich verdoppelt (Abb. 2B), wobei die Insel zwischen der dorsalen frontalo-parietalen KSG und der ventralen temporalen KSG liegt und von beiden mit radialen Gliafasern durch das Gliafaszikel in der Capsula externa mit Neuroblasten versorgt wird (Abb. 3). Dieses Gliafaszikel ist besonders prominent mit 15-18 SW (González-Arnay et al., 2017). Mit der Entwicklung des Temporallappens ist die Insel nicht länger der am weitesten lateral gelegene Kortexabschnitt, sondern verlagert sich 
ins Zentrum des Kortex. Diese neue, zentrale Lage dieser phylogenetisch alten Struktur ist im Einklang mit den integrativen Funktionen der menschlichen Insel, die sie zu einem wichtigen Knotenpunkt in einem neuronalen Netzwerk mit homöostatischen, emotionalen und kognitiven Aktivitäten machen.

Ein weiterer Aspekt der KSG als klar definierter Teil der Ventrikulärzone ist ihre Lobus-überschreitende Lage. Sie folgt longitudinal von vorn nach hinten der Grenze zwischen den zukünftigen Basalganglien und dem jeweils angrenzenden Kortex und reicht so von den präfrontalen Arealen durch frontale und parietale Areale bis in den Temporallappen hinein, wo die Ganglienhügel weniger ausgeprägt sind. Die jeweilige Lage der KSG in den verschiedenen Lobi lässt sich in Einklang bringen mit der Granularität der entsprechenden Inselregion, die von ihr entspringt. So könnte ein Zusammenhang zwischen dem agranulären Charakter der anterioren Insel mit dem agranulären Charakter der Frontalrinde bestehen, die der frontalen KSG benachbart ist. Die parietale KSG schließt sich ihrerseits an eine Ventrikulärzone an, die als Proliferationszone des somatosensorischen Kortex Ursprung eines extrem granulären Isokortex (von Economo, C. und Koskinas, G.N., 1925) ist. Entsprechend ist die dorsale und posteriore Insel des Menschen granulär und stellenweise sogar hypergranulär (Kurth, F. et al., 2010).

Interessanterweise ist das aufsteigende Gliafaszikel von der temporalen KSG in die Insel weniger stark ausgebildet, was mit den zwischen Insel und Temporalventrikel liegenden Fasertrakten (Commissura anterior) und Zellgruppen (Claustrum) zusammenhängen könnte. Diese Lagebeziehung und die damit verbundene erschwerte radiale Migration könnte den dysgranulären Charakter der mittleren und ventralen Insel erklären: Als Folge einer Vermischung von Migrationsströmen von Neuroblasten, die zum größeren Teil aus dem parietalen KSG, und zum geringerem Teil aus dem temporalen KSG einwandern.

Die Migrationsströme der menschlichen Insel wurden vor Kurzem mit „high-angular resolution diffusion magnetic resonance imaging“ Traktographie untersucht (Das,

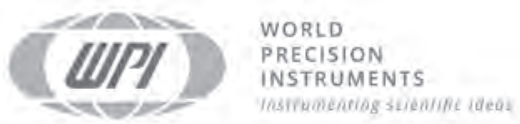

\section{Discover our new Motorised Stereotaxic Frames with our UMP3 injector}

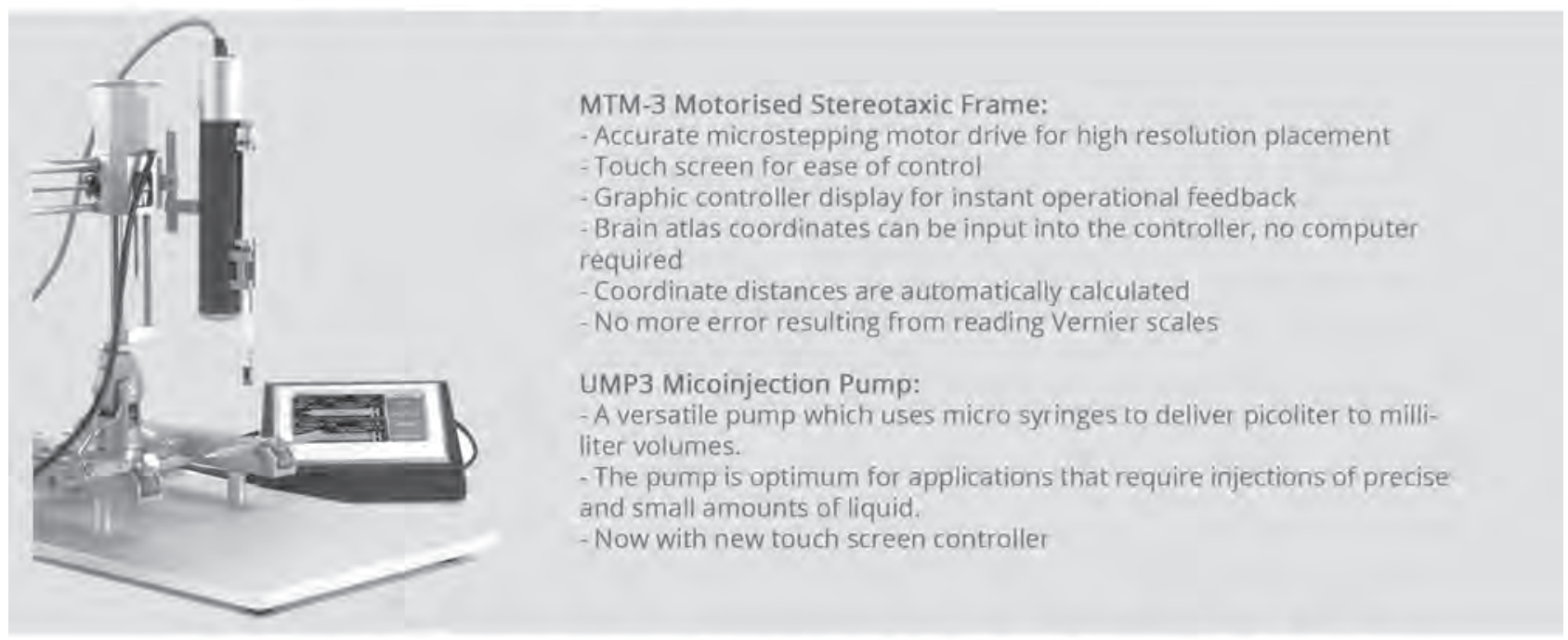




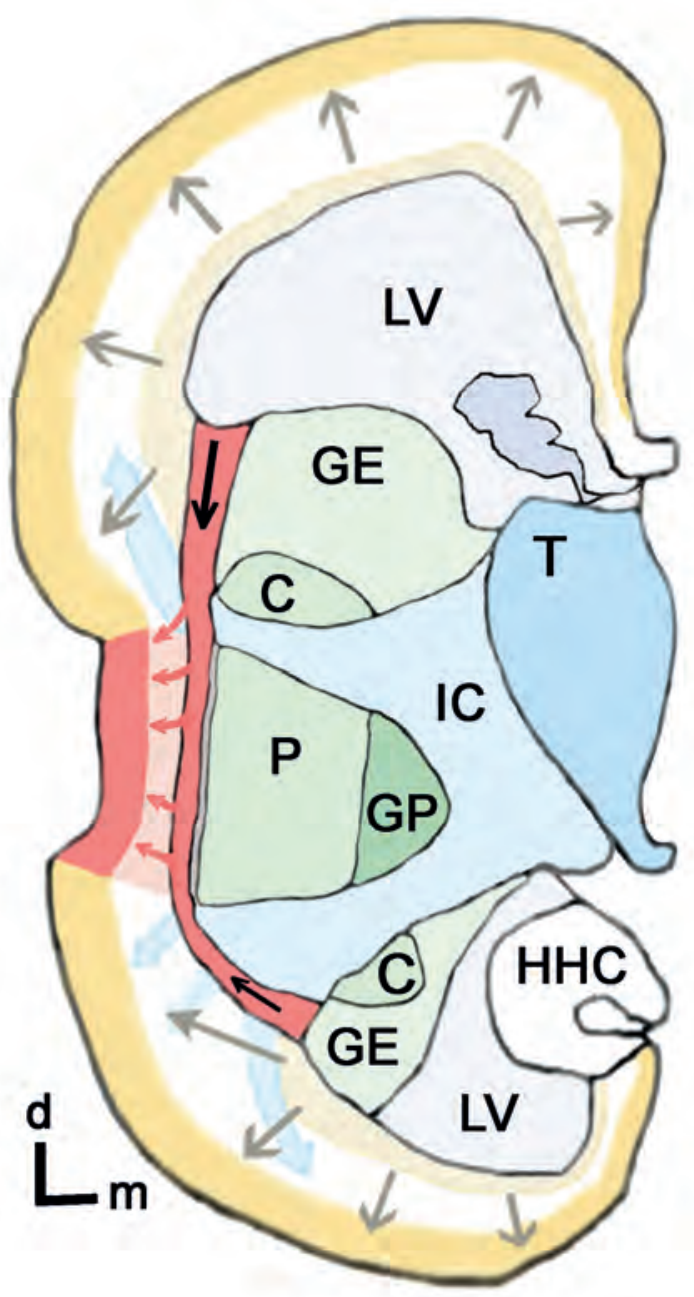

Abb. 3: Schematische Darstellung des Migrationsweges vom KSG des Frontal/Parietal- und Temporallappens in die Inselrinde (in rot), nach einem Coronalschnitt von einem 16 SW Fetus. Die Richtung der absteigenden und aufsteigenden Teile des radialen Gliafaszikels (auch in rot) ist mit Pfeilen gekennzeichnet. Die vorherrschende Orientierung der radialen Glia in den Hauptlobi ist mit grauen Pfeilen markiert. Die Capsula interna (IC) ist in Hellblau dargestellt; sie durchbricht die KSG auf ihrem Weg in die kortikale weiße Substanz. In Rosa: Subplate der Insel. C: Caudatus, GE: Ganglionäre Eminenz, GP: Globus pallidus, HHC: Kopf des Hippocampus, LV: Lateralventrikel, P: Putamen, T: Thalamus.

A. und Takahashi, E., 2017). Die Migrationsströme, die mit $15 \mathrm{SW}$ erkennbar sind, sind kompatibel mit dem von uns vorgeschlagenen Ursprung der Projektionszellen von der KSG und ihrer Migration entlang des radialen Gliafaszikels (González-Arnay, E. et al., 2017) (Abb. 3).

Das begrenzte Wachstum der Insel ist im Einklang mit dem begrenztem Abschnitt der Ventrikulärzone an der KSG, die als Ursprung der insulären Neurogenese und Migrationen identifiziert wurde. Ebenso wichtig für die Neurogenese des Kortex ist auch die wenig entwickelte Subvent- rikulärzone (SVZ) der Insel, deren Ausdehnung an der KSG von der Capsula interna begrenzt wird (González-Arnay et al., 2017). Die SVZ wird mittels Antikörperfärbungen gegen den Transkriptionsfaktor Tbr2 identifiziert (Betizeau, M. et al., 2013); sie wird in eine innere und äußere SVZ unterteilt, wobei letztere als Geburtsort vieler Neuronen der supragranulären Schichten III und II angesehen wird. Leider ist der Ursprung der Schicht IV im Menschen bis jetzt wenig erforscht; sie könnte von Progenitorzellen sowohl in der inneren als auch äußeren SVZ gebildet werden. Des Weiteren bildet die Insel keine eigene Intermediärzone aus, und auch die Subplate zwischen Insel und Capsula extrema ist viel schmaler als in den benachbarten Kortexgebieten. Von daher ist es bemerkenswert, dass die Insel in der Lage ist, eigene Gyri und Sulci auszubilden. Die Gyrierung der Insel läuft allerdings in einem späteren Zeitfenster $a b$, und zwar in den letzten Monaten der Schwangerschaft, wenn die neuronalen Migrationen weitgehend abgeschlossen sind (González-Arnay et al., 2017).

\section{Abschließender Kommentar}

Die Erforschung der Mechanismen, die zur architektonischen Differenzierung und zentralen Position der menschlichen Inselrinde führen, ist im Moment in den Anfängen. Experimentelle Strategien in Labortieren (z. B. genetische Modifikationen in der Maus) erscheinen wenig erfolgsversprechend angesichts der evolutionären Sonderstellung der menschlichen Insel. Wichtig ist auch der Umstand, dass viele Entwicklungsprozesse in der zweiten Hälfte der Schwangerschaft ablaufen, und damit im Labor experimentell (z. B. mithilfe von sogenannten „brain organoids“) kaum zu replizieren sind. Technische Verfeinerungen sowohl aktueller als auch die Entwicklung neuer bildgebender Methoden könnten helfen, die vorgeburtlichen Entwicklungsprozesse im Menschen weiter zu erforschen und besser zu verstehen, auch im Hinblick auf entwicklungsbedingte und neurodegenerative Pathologien.

So sind z. B. die agranulären Inselfelder stärker als die granulären Felder von fibrillären Ablagerungen in der Alzheimer-Krankheit betroffen (Bonthius, D.J. et al., 2005). Die Zahl der von Economo-Neuronen in der fronto-insulären Rinde ist in der frontotemporalen Demenz sehr stark reduziert (Santillo, A.F. et al., 2013). Bei SchizophreniePatienten wurde ein vermindertes Volumen insbesondere der anterioren Insel festgestellt (Shepherd, A. M. et al., 2012). Auch bei Autismus-Spektrum-Störungen fanden sich sehr komplexe Veränderungen der Inselaktivität, die 
in Unterfeldern in der rechten und linken Hemisphäre unterschiedlich ausfielen (Yamada, T. et al., 2016). Wie zu erwarten bei einem Hirngebiet wie der Inselrinde, die so eng mit Emotionswahrnehmung und affektiver Kontrolle verbunden ist, lassen sich auch in Patienten mit bipolaren affektiven Störungen vielfältige funktionelle und strukturelle Veränderungen nachweisen. So wurden z.B. ein vermindertes Volumen der grauen Substanz in der rechten hinteren und linken ventralen anterioren Insel, zugleich aber auch ein vergrößertes Volumen der grauen Substanz in der linken dorsalen anterioren Insel beschrieben (Tang, L.R. et al., 2014). Zusammengenommen zeigen diese Beispiele, dass die Insel in praktisch allen Fällen von psychischer Dysfunktion und psychiatrischen Krankheitsbildern in sehr verschiedener Art und Weise betroffen ist. Das unterschiedliche Verhalten der einzelnen Unterfelder der Insel ist besonders auffallend, und es stellt sich die Frage, wieweit die funktionellen Felder mit den zytoarchitektonischen Arealen identifiziert werden können. Die Inselrinde bleibt also weiterhin ein wenig verstandenes Kortexareal, und künftige Untersuchungen sollten ihre Komplexität in Struktur, Konnektivität und Entwicklungsgeschichte in Betracht ziehen.

Anmerkung: Die englische Version des Artikels ist online verfügbar unter https://doi.org/10.1515/nf-2018-A008

\section{Literaturverzeichnis}

Afif, A., Bouvier, R., Buenerd, A., Trouillas, J. and Mertens, P. (2007). Development of the human fetal insular cortex: study of the gyration from 13 to 28 gestational weeks. Brain Struct. Funct. 212, 335-346

Allen, G.V., Saper, C.B., Hurley, K.M. and Cechetto, D.F.(1991). Organization of visceral and limbic connections in the insular cortex of the rat. J. Comp. Neurol. 311, 1-16.

Allman, J.M., Tetreault, N.A., Hakeem, A.Y., Manaye, K.F., Semendeferi, K., Erwin, J.M. et al. (2011). The von Economo neurons in the frontoinsular and anterior cingulate cortex. Ann. N.Y. Acad. Sci. 1225, 59-71.

Arichi, T., Whitehead, K., Barone, G., Pressler, R., Padormo, F., Edwards, A.D. and Fabrizi, L. (2017). Localization of spontaneous bursting neuronal activity in the preterm human brain with simultaneous EEG-fMRI. Elife 6, pii e27814.

Assimacopoulos, S., Grove, E.A. and Ragsdale, C.W.(2003). Identification of a Pax6-dependent epidermal growth factor family signaling source at the lateral edge of the embryonic cerebral cortex. J. Neurosci. 23, 6399-6403

Augustine, J.R. (1996). Circuitry and functional aspects of the insular lobe in primates including humans. Brain Res. Brain Res. Rev. 22, 229-244

Bauernfeind, A.L., de Sousa, A.A., Avasthi, T., Dobson, S.D., Raghanti, M.A., Lewandowski, A.H. et al., (2013). A volumetric comparison of the insular cortex and its subregions in primates. J. Hum. Evol. 6, 263-279.

Betizeau, M., Cortay, V., Patti, D., Pfister, S., Gautier, E. et al.. (2013). Precursor diversity and complexity of lineage relationships in the outer subventricular zone of the primate. Neuron 80 , 442-457.

Bonthius, D.J., Solodkin, A. and Van Hoesen, G.W. (2005). Pathology of the insular cortex in Alzheimer disease depends on cortical architecture. J. Neuropathol. Exp. Neurol. 64, 910-922.

Buchanan, K.J. and Johnson, J.I. (2011). Diversity of spatial relationships of the claustrum and insula in branches of the mammalian radiation. Ann. N.Y. Acad. Sci. 1225 Suppl.1, E30-63.

Butti, C. and Hof, P.R. (2010). The insular cortex: a comparative perspective. Brain Struct. Funct. 214, 477-493.

Clascá, F., Llamas, A. and Reinoso-Suárez, F. (1997). Insular cortex and neighboring fields in the cat: a redefinition based on cortical microarchitecture and connections with the thalamus. J.Comp.Neurol. 384, 456-82

Craig, A.D. (2010). The sentient self. Brain Struct. Funct. 214, 563-577.

Craig, A.D. (2011). Significance of the insula for the evolution of human awareness of feelings from the body. Ann. N.Y. Acad. Sci. 1225, 72-82.

Das, A. and Takahashi, E. (2017). Neuronal Migration and Axonal Pathways Linked to Human Fetal Insular Development Revealed by Diffusion MR Tractography. Cereb. Cortex. 31, 1-9.

Fujita, S., Adachi, K., Koshikawa, N. and Kobayashi, M. (2010). Spatiotemporal dynamics of excitation in rat insular cortex: intrinsic corticocortical circuit regulates caudal-rostro excitatory propagation from the insular to frontal cortex. Neuroscience 165, 278-292.

Ghaziri, J., Tucholka, A., Girard, G., Houde, J.C., Boucher, O., Gilbert, G., Descoteaux, M., Lippé, S., Rainville, P. and Nguyen, D.K. (2017). The Corticocortical Structural Connectivity of the Human Insula. Cereb. Cortex 27, 1216-1228.

Ghaziri, J., Tucholka, A., Girard, G., Boucher, O., Houde, J.C., Descoteaux, M., Obaid, S., Gilbert, G., Rouleau, I. and Nguyen, D.K. (2018). Subcortical structural connectivity of insular subregions. Sci. Rep.8, 8596.

González-Arnay, E., González-Gómez, M. and Meyer, G. (2017). A Radial Glia Fascicle Leads Principal Neurons from the Pallial-Subpallial Boundary into the Developing Human Insula. Front. Neuroanat. 11, 111.

Kurth, F., Zilles, K., Fox, P.T., Laird, A.R. and Eickhoff, S.B. (2010). A link between the systems: functional differentiation and integration within the human insula revealed by meta-analysis. Brain Struct. Funct. 214, 519-534.

Medford, N. and Critchley, H.D. (2010) Conjoint activity of anterior insular and anterior cingulate cortex: awareness and response. Brain Struct. Funct. 214, 535-549.

Mesulam, M.M. and Mufson, E.J. (1985). The insula of Reil in man and monkey. Architectonics, connectivity, and function. In: Peters, A., Jones, E., editors. Cerebral cortex. New York, USA and London, UK: Plenum Publishing Corporation: p 179-224.

Molnár, Z. and Butler, A.B. (2002). The corticostriatal junction: a crucial region for forebrain development and evolution. Bioessays 24(6), 530-541.

Naidich, T.P., Kang, E., Fatterpekar, G.M., Delman, B.N., Gultekin, S.H., Wolfe, D., Ortiz, O., Yousry, I., Weismann, M. and Yousry, 
T.A. (2004). The insula: anatomic study and MR imaging display at 1.5 T. Am. J. Neuroradiol. 25, 222-232.

Nieuwenhuys, R. (2012). The insular cortex: a review. Prog. Brain Res. 195, 123-163.

Oppenheimer, S. and Cechetto, D. (2016). The Insular Cortex and the Regulation of Cardiac Function. Compr. Physiol. 6, 1081-133.

Puelles, L., Kuwana, E., Puelles, E., Bulfone, A., Shimamura, K., Keleher, J. et al., (2000). Pallial and subpallial derivatives in the embryonic chick and mouse telencephalon, traced by the expression of the genes Dlx-2, Emx-1, Nkx-2.1, Pax-6, and Tbr-1. J. Comp. Neurol. 424, 409-438.

Rakic, P. (1974). Neurons in rhesus monkey visual cortex: systematic relation between time of origin and eventual disposition. Science 183, 425-427.

Santillo, A.F., Nilsson, C. and Englund, E. (2013). von Economo neurones are selectively targeted in frontotemporal dementia. Neuropathol. Appl. Neurobiol. 39(5), 572-9.

Shepherd, A.M., Matheson, S.L., Laurens, K.R., Carr, V.J. and Green, M.J. (2012). Systematic meta-analysis of insula volume in schizophrenia. Biol. Psychiatry 72, 775-784.

Singer, T., Seymour, B, O’Doherty, J., Kaube, H., Dolan, R.J. and Frith, C.D. (2004). Empathy for pain involves the affective but not sensory components of pain. Science 303, 1157-1162.

Tang, L.R., Liu, C.H., Jing, B., Ma, X., Li, H.Y., Zhang, Y., Li, F., Wang, Y.P., Yang, Z. and Wang, C.Y. (2014). Voxel-based morphometry study of the insular cortex in bipolar depression. Psychiatry Res. 224(2), 89-95.

Tanriover, N., Rhoton, A.L, Jr, Kawashima, M., Ulm, A.J. and Yasuda, A. (2004). Microsurgical anatomy of the insula and the sylvian fissure. J. Neurosur. 100, 891-922.

Von Economo, C. and Koskinas, G.N. Die Cytoarchitektonik der Hirnrinde des erwachsenen Menschen. J. Springer Berlin (1925).

Yamada, T., Itahashi, T., Nakamura, M., Watanabe, H., Kuroda, M., Ohta, H., Kanai, C., Kato, N. and Hashimoto, R.I (2016). Altered functional organization within the insular cortex in adult males with high-functioning autism spectrum disorder: evidence from connectivity-based parcellation. Mol. Autism 5; 41.

Zilles, K., Zilles,B. and Schleicher, A. (1980). A quantitative approach to cytoarchitectonics. VI. The areal pattern of the cortex of the albino rat. Anat. Embryol. (Berl) 159, 335-360

\section{Autoreninformation}

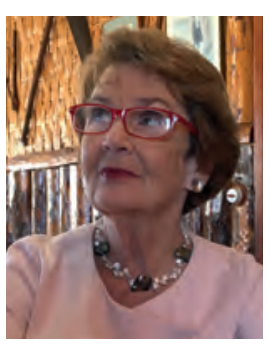

Gundela Meyer

Facultad de Medicina, Universidad de La Laguna

Tenerife

Spain

E-Mail: gundelam@aol.com

Prof. Dr. med. em. Gundela Meyer studierte Medizin an den Universitäten Göttingen und Valencia (Spanien). Sie promovierte an der Universität La Laguna (Spanien). Es folgte die Facharztausbildung in Psychiatrie und der Ruf auf die Professur für Neuroanatomie an der Universität La Laguna. Hauptthema ihrer Forschung ist die Entwicklung der Großhirnrinde, inbesondere der des Menschen. 